\title{
Screening to Identify the Unrecognized Victims in Practice: Implementing an EBP HITS Domestic Violence Tool in Neurology
}

\author{
MaryAnn Martin* \\ School of Nursing, Graceland University, USA
} *Corresponding author: MaryAnn Martin, School of Nursing, Graceland
University, Kansas City, USA.
Received Date: September 23, 2020

Published Date: October 23, 2020

\begin{abstract}
Domestic violence (DV) or intimate partner violence (IPV) is the cause of major physical and emotional health consequences in neurological patients; yet many are not screened or referred for DV services. A need for the DV screening was identified for this project since the neurology practice did not have a DV screening policy and limited research for screening in neurology practices is in the literature. This pilot quality improvement (QI) study occurred in a 3-office private neurological practice in South Florida that treated 300 to 400 patients per month. The problems associated with the complications of DV among neurological patients can create increased psychological and chronic pain conditions while also leading to reduced quality of life from the sequalae related to the DV trauma. This QI quasi-experimental study aimed to evaluate the Nurse Practitioner (NP) provider screening and referral rates pre and post educational intervention using the evidence-based practice (EBP) HITS DV screening tool (Hurts, Insults, Threatens, Screams). The secondary objectives of the project were to translate this to screening practice by educating the staff on the evidenced based DV training and implementing an organizational policy for universal domestic violence screening. The Ace Star model of evidence-based practice was utilized as the conceptual framework (Permission for use was obtained via email from the author Dr. Kathleen Stevens). The ACE framework offered the 5 steps to evaluate and implement effective practice and organizational policy change. Final results of the study indicated an increase in provider compliance post educational intervention with DV screening and referral rates. The research questions were answered with further data analysis demonstrating an increase in provider DV screening and referrals with pre and post percentage scores. Scores ranged from $0.3 \%$ pre intervention screened and $87 \%$ patients screened post intervention. DV referrals pre intervention were $0.6 \%$ and post referral were $100 \%$ with the 10 patients that screened positive in the one-month time period. Results were consistent with other literature indicating provider screening barriers and more women screened positive on the HITS tool with 8 women and 2 men out of 10 positive screens in the one-month time period.
\end{abstract}

Keywords: Domestic Violence; Intimate Partner Violence; HITS DV screening tool; neurological complications; ACE Star model.

\section{Introduction}

The current gap in literature indicates that only $10 \%$ of health care providers are currently screening for domestic violence (DV) in practice [1,2]. DV has been shown to occur across genders, races, and economic classes and is not only against women with a $50 \%$ occurrence between genders in some cases [3]. DV is a sensitive topic to discuss for both the provider and the victim. Domestic violence and intimate partner violence (IPV) terms are often used interchangeably. Literature has indicated that it takes time for the victim to open up about the domestic violence experiences. As a result, the problem is often missed by providers or takes multiple visits to be recognized by the provider or for the patient to feel comfortable to divulge this sensitive information to the provider. Chapin, et al. (2011) [2] study evaluated provider self-efficacy and confidence with education in DV screening and indicated that education was an important factor for medical providers to be more confident advocates for DV screening. Currently, DV screening policies vary among health care organizations and providers. Currently, DV screening is predominantly performed in Emergency 
Departments but DV is also seen in neurological patients sustaining injuries. Neurological patients present with head trauma and spinal injuries but literature indicates that these patients are not being screened and identified in this specific practice. These injuries can lead to continued problems and complications that can exist well past the abuse and trauma with contribution to other health conditions like seizures, cardiac problems, gastrointestinal problems, decreased memory and cognition, and migraines headaches [4,5]. The HITS domestic violence screening tool developed by Dr. Kevin Sherin et al. (1998) [6] has been identified as a short, efficient and highly sensitive screening tool with a Cronbach alpha of 0.80 . The HITS tool can be implemented into a practice to help screen and identify neurological patients (victims) potentially experiencing DV. The HITS DV screening tool stands for acronym (HURTS, INSULT, THREATEN, and SCREAM) that is verbally given to the patient and scored based on a five-point Likert scale: never, rarely, sometimes, fairly often, and frequently [6]. The answer for each question is given 1 point ranging up to 5 points and the scores can range from 4 to 20 . A positive screen is considered with a score of 10 or above. If the screen is positive, it is recommended that the provider further discuss the score with the patient and determine if the patient is suffering from DV abuse an offer resources and services to meet the patient's needs [6].

\section{PICOT}

The quality improvement project used the PICOT (population, intervention, comparison, outcomes, and time) framework to guide the project. The PICOT question was the following: In a neurology practice treating adult patients over the age of $18(\mathrm{P})$, does an educational intervention implementing the HITS domestic violence screening tool (I) versus usual care (C) have an impact on Nurse Practitioner's (NP's) screening and referral practices $(0)$ over a 1-month time period (T)?

\section{Research Questions}

- Question \#1: Does implementation of the HITS DV screening tool influence Nurse Practitioners screening practices?

- Question \#2: Does implementation of the HITS DV screening tool influence Nurse Practitioners referral practices?

\section{Objectives}

The study objectives were to evaluate the impact of screening and referral rates of a nurse practitioner after education and universal implementation of the HITS DV screening tool in a neurological practice.

\section{Conceptual Framework}

The main focus of this QI project was to identify patients experiencing domestic violence via utilization of the HITS DV tool. It was essential for the provider to understand the importance of recognizing and identifying victims of DV so these victims could be referred to appropriate services in the community. The conceptual framework selected for this project was the ACE star model developed by Kathleen Stevens (2004) at the University of Texas Health Sciences at San Antonio. This study utilized all of the ACE star model 5-star points to demonstrate knowledge transformation at the following stages:

- Discovery Research: Neurological practice did not have a DV screening policy. It was necessary to gain approval and consent from the administration and organization for the project.

- $\quad$ Evidence Summary: Literature review identified a gap in DV screening in practice with limited literature identified for screening in neurological practices. Gained approval from IRB. Gained approval from Dr. Sherin to utilize the HITS DV screening tool via email. Gained approval from Dr. Stevens to utilize the ACE star model.

- Translation to guidelines: Reviewed USPTSF (2013) guidelines for Domestic violence recommendations. Implemented universal screening into the Neurology practice with translation to practice with final implementation of a DV screening policy.

- Practice Integration: Educate the staff on DV, gather the pre and post knowledge data from staff using evidence-based education, implement universal screening process using the HITS DV screening tool.

- Process and Outcome evaluation: Gather 1-month retrospective patient data pre education and intervention, implement project, check reliability of HITS scoring with staff, periodic checks of universal screening process, at 1-month completion of project gather and evaluate NP screening and referral data retrospectively in the electronic medical record (EMR). Analyze the collected data for demographic characteristics and provider DV screening and referral rates. Deliver the executive summary to the project organization and develop the policy change for DV screening in practice to ensure sustainability plan [7].

\section{Design, Setting, Sample and IRB approval}

The study was implemented in a 3-office privately owned neurology practice in South Florida and permission was obtained for the project from the administration. The Neurology practice treated a variety of patients ranging from Multiple Sclerosis, Epilepsy, Stroke, head and neck injuries, and Alzheimer's. On a monthly basis approximately 300-400 patients were seen by the Nurse Practitioner (NP) provider. All staff voluntarily agreed to participate in the QI project. Universal screening using the HITS DV screening tool was implemented in the clinic to ensure that all patients over the age of 18 received the same screening process, informed consent, and screening information. Private screening guideline recommendations were followed according to the US 
Preventative Services Task Force (USPTSF) recommendations (2013) [8] and patients were given information on the universal screening process prior to receiving the universal DV screening tool. If patients declined to be screened, or they wanted their family member in the room the patient was not screened and were excluded from the project. Institutional Review Board (IRB) approval was received for this project.

\section{Educational Intervention}

Prior to the start of the project, all staff that worked directly with patients were given the DV education training. Education on Domestic violence included the following items: recognizing DV, the cycle of DV, information on the HITS screening tool, education on the scoring of the HITS tool, and referral recommendations. If the HITS screening scores were 10 or greater (positive screens), information on where to refer and available DV services in the community and nationally were provided to the staff and NP to give to the patients. Detailed information on private screening and screening recommendations were also provided in the educational training.

\section{Project Implementation}

A 1-month retrospective chart review was performed on 322 charts. The post implementation project was performed over a period of 1 month after the education was provided. Reliability and validity were maintained during the screening process with verification of the HITS scoring on 5 of the first day patients by the provider and the project investigator to compare screening scoring. At completion of the 4 weeks, 300 retrospective charts were reviewed for documentation of the universal HITS screening tools, positive screens, and documented provider referrals. The same data collection form was used to gather the NP provider screening and referral rates and demographic information only on age and gender of the referred and screened.

\section{Data Analysis}

A total of $263 \mathrm{DV}$ patients screened out of the 300 total patients resulted in an $87 \%$ screening rate post intervention in the onemonth universal DV screening period. These results were compared to pre intervention DV screening rates with results showing 0.31 percent out of 322 retrospective charts reviewed with no universal screening policy in place at the office. Out of the 263 DV screened patients, 28 patients declined to be screened, which was $9 \%$ that were offered screening by the provider. The remainder of the patients were not screened for various reasons mostly related to barriers with the providers schedule being too busy. A total of 10 patients screened positive post intervention and were referred which indicated a 4.2 percent of the 235 patients actually screened. This indicated a significant increase from the 0.31 percent screened and 0.62 percent referred in the pre intervention retrospective chart review.

\section{Limitations}

Several limitations were identified in this pilot study. The actual sample size and provider sample size was small. It would be recommended to perform this study with more providers of varying licenses ranging from: Nurse Practitioner, Medical Doctor, and Physician Assistant. The 1-month retrospective pre and post chart review lead to small patient chart data set so randomization could not be implemented on the chart review. As a result, all charts were reviewed. It would be recommended for a longer duration for the retrospective chart review over at least a 6 months study time period to improve the significance of the study. Further evaluation on barriers to screening with an attitudes and barriers survey should be performed since provider time constraints were evident with lack of universal screening on all patients seen during the 1-month intervention. In addition, 9 percent of the convenience sample population declined to be screened and reason for the decline was not evaluated and should be further evaluated in another study. Provider time constraints in screening has been indicated in other studies as a significant barrier [9].

\section{Conclusion}

Literature gaps currently exist in regards to evaluating patients experiencing domestic violence in the neurological setting with evidenced based practice screening. The study provides findings showing a significant percentage increase in NP provider domestic violence screening and referral rates in the neurological practice post educational intervention and utilization of the HITS domestic violence screening tool. Screening in the neurology practice may be able to make a difference for many neurological patients living as unrecognized domestic violence victims. Screening and identification can help improve patient outcomes in this higher risk population group.

\section{Acknowledgement}

I am thankful for my chair and mentors' support and guidance in this project.

\section{Conflict of Interest}

Author does not have any conflict of interest. No outside funding received for this project.

\section{References}

1. Bonds D, Ellis S, Weeks E, Palla S, Lichstein P (2013) A practice centered intervention to increase screening for domestic violence in primary care practices. BMC Family Practice.

2. Chapin J, Coleman G, Varner E (2011) Yes, we can! Improving medical screening for intimate partner violence through self-efficacy. J Inj Violence Res 3(1): 19-23.

3. Patra P, Prakash J, Patra B, Khanna P (2018) Intimate partner violence: Wounds are deeper. Indian J Psychiatry 60(4): 494-498.

4. Siemieniuk R, Krentz H, Gish J, Gill J (2010) Domestic violence screening: Prevalence and outcomes in a Canadian HIV population. AIDS Patient Care and STDs 24(12): 763-770. 
5. Campbell J, Anderson J, McFadgion A, Gill J, Zink E, et al. (2018) The effects of intimate partner violence and probable traumatic brain injury on central nervous system symptoms. J Womens Health (Larchmt) 27(6): 761-767.

6. Sherin K, Sinacore J, Li X, Zitter R, Shakil A (1998) HITS: A short domestic violence screening tool for use in a family practice setting. Family Medicine 30(7): 508-512.

7. Stevens K (2013) The impact of evidence-based practice in nursing and the next big ideas. Online J Issues Nurs 18(2): 4.
8. US Preventative Services Task Force [USPSTF] (2013) Screening for intimate partner violence and abuse of elderly and vulnerable adults: U.S. preventive services task force recommendation statement 158(6): 478-486.

9. DeBoer M, Kothari R, Kothari C, Koestner A, Rohs T (2013) What are the barriers to nurses screening for intimate partner violence? J Trauma Nurs 20(3): 155-160. 